\title{
Le masque dans les comédies de Richard Brome, un divertissement sous influence continentale?
}

\section{Claire Bardelmann}

\section{(2) OpenEdition}

\section{Journals}

\section{Édition électronique}

URL : http://journals.openedition.org/shakespeare/737

DOI : $10.4000 /$ shakespeare.737

ISSN : 2271-6424

Éditeur

Société Française Shakespeare

\section{Édition imprimée}

Date de publication : 1 novembre 2005

Pagination : 13-25

ISBN : 2-9521475-1-5

\section{Référence électronique}

Claire Bardelmann, «Le masque dans les comédies de Richard Brome, un divertissement sous

influence continentale? », Actes des congrès de la Société française Shakespeare [En ligne], 22 | 2005, mis en ligne le 01 janvier 2007, consulté le 01 mai 2019. URL : http://journals.openedition.org/ shakespeare/737 ; DOI : 10.4000/shakespeare.737 


\section{Shakespeare et l'Europe de la Renaissance}

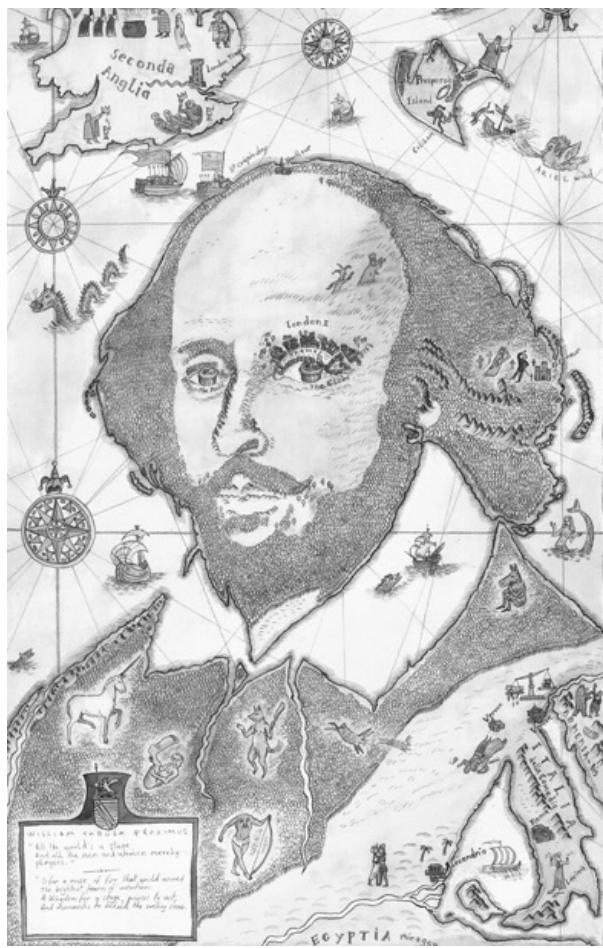

a ctes du Congrès

organisé par la

SOCIÉTÉ FRANÇAISE SHAKESPEARE

les 11,12 et 13 mars 2004

textes réunis par

Pierre KAPITANIAK

sous la direction de

Yves PEYRÉ 


\title{
COMITÉ SCIENTIFIQUE :
}

\author{
Margaret Jones-Davis \\ Jean-Marie Maguin \\ Yves Peyré \\ Pierre Kapitaniak
}

COUVERTURE :

Edouard Lekston, William Tabula Proximus, 2004

conception graphique et logo

Pierre Kapitaniak

CC 2004 Société Française Shakespeare

Institut du Monde Anglophone

Université de Paris III - Sorbonne Nouvelle

http:// univ-montp3.fr/SFS/

5 rue de l’École de Médecine

75006 Paris

ISBN 2-952 1475-1-5

Tous droits de traduction, de reproduction et d'adaptation réservés pour tous les pays 


\title{
LE MASQUE DANS LES COMÉDIES DE RICHARD B ROME, UN DIVERTISSEMENT SOUS INFLUENCE CONTINENTALE?
}

\author{
Claire BARDELMANN
}

\begin{abstract}
Sous l'influence du ballet de cour français, le masque caroléen subit de profondes transformations, qui montrent l'érosion de l'idéal jonsonien, supplanté par la recherche du spectaculaire. L'antimasque tel qu'il avait été introduit par Jonson est dépouillé de sa fonction dramatique, tandis que sont massivement introduites les très descriptives entrées à la française. La métamorphose du masque de cour caroléen se reflète dans le théâtre contemporain. Changes, de James Shirley, critique sévèrement le masque nouvelle mode, tandis que The English Moor, comédie de Richard Brome, utilise des entrées à la française. Dans The Antipodes, la structure du masque imprègne celle de la pièce. Cependant, et en dépit des modifications apportées par l'influence française, les œuvres de Richard Brome utilisent les éléments du masque à des fins dramatiques, fidèles en cela au principe jonsonien d'union de la musique et du théâtre. Le jeu des influences annonce ainsi les nouvelles formes dramatico-musicales qui se développent à la Restauration.
\end{abstract}

Under the influence of the French "ballet de cour", the Carolean masque undergoes deep structural transformations showing a shift from the Jonsonian concern for dramatic interest to an increasing emphasis on the visual and spectacular dimension of the masque. The Jonsonian antimasque tends to lose its dramatic function, and descriptive "entrées à la française" become the rule. The metamorphosis of the Carolean courtly masque is reflected in contemporary drama, as some plays by James Shirley and Richard Brome show. In Shirley's Changes, the new features of the masque come under sharp criticism, while Brome's comedy The English Moore uses entries after the French manner. In The Antipodes, the structure of the masque permeates the dramatic pattern of the play. However, in Richard Brome's drama, the use of masque elements retains the Jonsonian principle of union between music and drama, thus announcing the rise of hybrid dramatic forms after the Restoration.

J e m 'intéresse aujourd'hui aux rapports entre masque et théâtre à l'époque caroléenne sous un angle particulier, celui de l'influence qu'a pu exercer le ballet de cour français de la même période à travers l'exemple d'un dramaturge, Richard Brome.

Cette étude part d'un constat : dans le théâtre caroléen se trouve beaucoup de musique, surtout dans les comédies de Richard Brome et de James Shirley. Les masques ou éléments de masques y occupent une place particulièrement importante. Parallèlement, le masque de cour lui-même subit des modifications en profondeur sous l'influence musicale étrangère, c'est-à-dire très prioritairement française.

On peut donc se demander en quoi le masque caroléen reflète cette influence française - en l'occurrence, celle du ballet de cour - et si le théâtre s'en faisait l'écho, non seulement à travers des allusions aux pratiques musicales de l'époque, mais aussi dans les éléments de masque que l'on peut trouver à la scène, surtout chez Richard Brome, 
dont le théâtre est particulièrement imprégné de musique. En effet, cette densité musicale, ainsi que la présence de masques dans certaines pièces, suggèrent une hybridation des formes qui reflète aussi les genres à la mode, et donc, potentiellement, les nouvelles orientations du masque de cour. Les dramaturges ont-ils répercuté ces changements structurels dans les masques insérés au théâtre, et comment l'influence française influe-t-elle sur la dynamique des genres?

Mon but est donc d'étudier les changements structurels du masque caroléen et de voir s'ils se retrouvent au théâtre, et dans quelle mesure ils influent sur la structure dramatique. Je commencerai donc par établir l'influence du ballet de cour sur le masque anglais sous Charles ${ }^{\text {er }}$. Puis, j'essaierai de déterminer la place de cette influence dans l'interaction du masque de cour et du théâtre, principalement à travers deux comédies de Richard Brome (The English Moor et The Antipodes).

\section{Ballet de cour et masque}

L'influence française sur le masque caroléen est liée à la forte présence musicale française sous Charles I ${ }^{\text {er }}$. En fait, cette place remonte au début du XVII ${ }^{\mathrm{e}}$ siècle. Ainsi, le maître à danser du prince Henri était-il français (Nicolas Villiard). Jacques Cordier, surnommé «Bochan», violoniste et maître à danser, était membre de la maison du prince Henri en 1608, et recevait deux fois le salaire des violonistes du Roi (les vingt-quatre violons du Roi, d'inspiration française, étaient appelés «les disciples de Bochan»), eut le même statut privilégié dans les premières années du règne de Charles ${ }^{\text {er }}$. La reine Henriette-Marie avait aussi ses musiciens français, comme Nicolas Picard, membre de la musique du Roi pendant toute la durée du règne.

Sous l'influence de la Reine, ces musiciens apportent à l'Angleterre une expérience de première main du ballet de cour français. Henriette-Marie elle-même avait participé à des ballets de cour avant de partir pour l'Angleterre (en 1623, elle dansait dans Les Fêtes de Junon; en 1621, elle représentait Aurore dans le Grand Ballet de la Reyne représentant le Soleil. À la Cour, les maîtres à danser français contribuent à l'organisation des masques - Bochan, par 
exemple, mit la main aux danses de deux masques, Love Freed et The Lord's Masque.

Ce rôle des maîtres à danser dans l'importation du ballet de cour apparaît clairement dans une comédie de William Cavendish, The Variety (représentée au théâtre des Blackfriars, ce qui la date d'avant 1642). La comédie se moque des modes françaises en général et des maîtres à danser en particulier, à travers le personnage de Galliard, un maître à danser qui participe à l'élaboration d'un masque. La conception du masque selon Galliard fait l'objet d'une vive critique ; à travers ce personnage, le dramaturge déplore l'absence d'intérêt du texte, le caractère décousu de l'intrigue, l'accent mis sur le caractère spectaculaire du divertissement au détriment du reste :

GALLIARD. Aw, de write? dat is noting alamode, your speesh two, tre, yard long, pfaw ? give a me de quick a spirit, de fancie, de brave scene, de varietie of de Antimasque, de nimble a foot, no matter de sense, begar it vole be de brave ting ${ }^{1}$.

Cette description d'un bon masque selon Galliard est très française «the brave scene» et «the varietie of the Antimasque» étant des références précises et directes aux modifications subies par le masque sous l'influence du ballet de cour français.

La critique formulée par Cavendish renvoie à en effet un constat: le masque anglais, sous Charles $\mathrm{I}^{\mathrm{er}}$, importe un modèle déjà décadent en France, celui du ballet à entrées. Le ballet à entrées est un type de ballet différent de ceux qui le précèdent (ballet comique, ballet mélodramatique), car il ne présente pas d'unité dramatique. À l'origine, le ballet de cour (introduit en France à la fin du XVI ${ }^{\mathrm{e}}$ siècle), traduit un désir de retrouver la formule du drame antique, avec une union harmonieuse de la poésie, de la musique et de la danse. Quel que soit le sujet, le ballet de cour a une trame dramatique suivie, une unité empruntée au drame; la musique et la danse participent à l'action. Cette ambition disparaît précisément avec le ballet à entrées, vers 1620 .

Le plan du ballet à entrées inspiré du ballet mascarade est très simple : un récit initial, suivi d'un nombre variable d'entrées dansées mais sans chant (des pantomimes, les personnages s'expriment par gestes plus que par chants), puis d'un chœur qui annonce en chantant

\footnotetext{
${ }^{1}$ W. Cavendish, The Variety (1649), II.ii.44-47.
} 
la venue des danseurs du grand ballet, la danse finale et la seule à laquelle participent les nobles de la Cour.

Le Ballet des mariages sans degoust et sans cocuage (1638) offre un exemple de ce type de ballet. Le récit chanté par Hymen est suivi de cinq entrées puis du grand ballet sous la forme d'une «Bouffonnerie dansée en salade », selon le plan suivant :
Récit chanté par Hymen
Première entrée dansée par le Goust
Seconde entrée dansée par la saucisse et la moutarde
Troisiesme entrée dansée par l'orange et la perdrix
Quatriesme entrée dansée par le jambon et la bouteille
Cinquiesme entrée dansée par la poire et le fromage
Grande bouffonnerie dansée en salade ${ }^{2}$.

Dans ce type de ballet, les personnages entrent arbitrairement les uns après les autres; l'énumération des scènes suffit à exposer le sujet du spectacle, sans recours au texte. Les entrées ont toujours un caractère expressif, mais descriptif ; elles forment la comédie muette du ballet de cour. Comme l'exprime l'abbé De Pure, « les gestes et les mouvements signifient ce qu'on pourroit exprimer par des paroles ${ }^{3} »$.

Le ballet à entrées, qui sacrifie ainsi l'intrigue au spectaculaire, constitue une période de régression dans le développement du ballet de cour. Or, c'est précisément la forme qui influence le masque caroléen.

L'influence du ballet de cour sur le masque anglais est principalement structurelle. Elle concerne surtout le changement de statut de l'antimasque jonsonien, sous l'influence des entrées à la française (la « varietie of Antimasque » à laquelle fait allusion le maître à danser Galliard); et l'importance croissante du spectaculaire au détriment de l'intérêt dramatique. Dans ses grandes lignes, l'évolution de masque suit donc celle du ballet de cour, dont elle importe le modèle.

La structure du masque est d'abord modifiée par le changement de fonction de l'antimasque. À l'origine, la fonction de l'antimasque (introduit en 1611 dans Oberon de Ben Jonson) est de divertir mais

\footnotetext{
${ }^{2}$ Dans P. Lacroix (éd.), Ballets et mascarades de cour de Henri III à Louis XIV, Genève, Slatkine Reprints, 1968, vol. 5.

${ }^{3}$ De Pure, Idée des spectacles anciens et nouveaux, cité dans H. Prunières, Le Ballet de cour en France avant Benserade et Lully, Plan-de-la-Tour, Éditions d'Aujourd'hui, 1983, p. 168.
} 
aussi d'introduire un contraste avec la trame sérieuse du masque. Elle précède la principale danse des «masquers», la «masquing dance» présentée par le roi et ses seigneurs, ou par la reine et ses dames; par contraste, elle en rehausse la noblesse. Sous Charles ${ }^{\mathrm{er}}$, l'antimasque perd cette fonction dramatique voulue par Ben Jonson. Le nombre des antimasques augmente et cette partie du divertissement gagne simultanément en volume à l'intérieur du masque. En outre, les différents tableaux de l'antimasque ne sont plus reliés entre eux ni à la trame principale, ce qui en amoindrit l'intérêt dramatique au profit du spectacle visuel.

En fait, l'antimasque devient peu à peu une succession d'entrées à la française : de longues séries de danses (ou de pantomimes, car il ne s'agit pas de danses de cour) non séparées par des chansons ou des récits.

C'est à partir des années 1630 que cette influence se fait sentir le plus vivement. À partir de Chloridia (1631) de Ben Jonson et Inigo Jones, l'antimasque devient intégralement dansé. Les désastres causés par Cupidon dans ce masque sont illustrés par une série de danses ininterrompue, numérotées de 1 à 8 et introduites par un récit déclamé et une danse d'un postillon nain; ce morceau est appelé «the first entry of the antimasque», et les entrées suivantes «second Entry», etc. C'est aussi la première utilisation du terme «entry » appliqué à l'antimasque, pour décrire des épisodes pratiquement identiques aux entrées du ballet de cour français : les personnages entrent, dansent, s'en vont. Le lien entre les danses est lâche, et l'élément de pantomime est important, comme en témoigne la description de la troisième entrée :

3 Entry

The Queenes Dwarfe, richly apparel'd, as a prince of hell, attended by six infernall Spirits. He first danceth alone, and then the Spirits : all expressing their joy, for Cupids comming along them ${ }^{4}$.

Par la suite, cette utilisation des entrées pour structurer l'antimasque devient habituelle, et même, à partir de 1630, indispensable, comme l'indique un dialogue de The Triumph of Peace (1634):

\footnotetext{
${ }^{4}$ Dans D. Linley, Court Masques: Jacobean and Caroline entertainments 1605-1640, Oxford, O.U.P., 1995, p. 234.
} 
FANCY. How many antimasques have they? of what nature?

For these are fancies that take most; your dull

And phlegmatic inventions are exploded.

Give me a nimble antimasque ${ }^{5}$.

De fait, suit une série d'antimasques où l'on retrouve des pantomimes à la française, et même des acrobaties dans la même veine : antimasque de Fancy, Novelty et Admiration, «the dance expressing the natures of the presenters ${ }^{6} »$. Puis des entrées se succèdent, dont le thème est simplement d'illustrer toutes les formes que peut prendre la fantaisie de l'imagination, ce qui est prétexte à moult changements de décor. Les personnages, dans leur dialogue, ne font que commenter le changement de tableaux. Interviennent ainsi un second antimasque composé de différents personnages qui fréquentent une taverne (mère maquerelle, serviteurs, gentilhomme, mendiants); puis, une série d'entrées, même si elles ne portent pas ce nom : un paysan, un érudit, un médecin ; puis, nouveau changement de décor et entrées d'oiseaux : hibou, corneille, faucon, pie, geai, puis des personnages divers : voleurs et marchands; chasseurs, nymphes et satyres.

Cette adoption progressive, mais massive, des entrées à la française a un lien direct avec un autre changement structurel du masque caroléen, à savoir la montée en puissance de l'aspect visuel et le déclin de l'intérêt dramatique.

Il existe très certainement un lien entre l'accent mis en France sur la splendeur visuelle du masque et la fascination pour les arts visuels sous Charles $\mathrm{I}^{\mathrm{er}}$. Inigo Jones (connu pour son association avec Ben Jonson, et qui conçut aussi les décors de nombreux masques caroléens) était très intéressé par les divertissements de cour étrangers et notamment français, et par l'architecture française (il remodela une partie de Somerset House pour la reine, en collaboration avec des architectes français). On connaît aussi sa rivalité et son désaccord avec Ben Jonson. Jonson voulait conserver au masque son contenu littéraire, Inigo Jones ne voulait rien qui interfère avec la splendeur de la mise en scène, à laquelle il voulait donner la première place, et Jonson dut s'effacer à partir de 1631, au profit de librettistes... francophiles, qui avaient davantage la faveur de la reine, comme Aurelian Townshend, Thomas Carew (l'auteur de Coelum

5 J. Shirley, The Triumph of Peace, in English Masques, Londres, Blackie and Sons, p. 211.

6 Ibid., p. 219. 
Britannicum), mais aussi Shirley et Davenant ${ }^{7}$. Le coût des masques reflète l'accent mis sur le spectaculaire: le plus onéreux de tous les masques caroléens, à savoir The Triumph of Peace de Shirley, avec musique de William Lawes et Simon Ives, coûta plus de 21000 livres, (l'équivalent de plus d'un million d'euros). Cette splendeur est d'ailleurs soulignée à la fin du livret :

And thus concluded this Masque, which was, for the variety of the shows, and richness of the habits, the most magnificent that hath been brought to court in our time ${ }^{8}$.

L'accent mis par Inigo Jones sur une mise en scène spectaculaire suit très exactement l'évolution du ballet de cour en France, et elle va bien de pair avec l'adoption des entrées à la française, sans véritable fil directeur mais qui permettait un grand déploiement de costumes, décors, etc. Cette tendance culmine avec Salmacida Spolia, dernier des masques caroléens (1640), et où l'on ne trouve pas moins de vingt entrées. On y voit que les entrées sont aussi prétexte à déployer une dimension visuelle spectaculaire, ce que le livret indique clairement :

2. Entry

Four old men richly attired, the shapes proper to the persons

[...]

3. Entry

Three young soldiers in several fashioned habits, but costly

[...]

14. Entry

An amorous courtier, richly apparelled ${ }^{9}$.

De plus, les décors spectaculaires se succèdent dans ce masque : scène de tempête avec sphère qui s'enflamme pour révéler une Furie, décor champêtre avec descente d'un char argenté portant la Concorde, puis, après les antimasques (entrées), décor de montagnes inaccessibles et, à la fin, descente d'un nuage multicolore portant la Reine et ses suivantes, vêtues de costumes magnifiques :

in Amazonian habits of carnation, embroidered with silver, wilth plumed helms, baudrickes with antique swords hanging by their sides,

\footnotetext{
${ }^{7}$ Voir O. Gombosi, «Some musical aspects of the English Court Masque », Journal of the American Musicological Society, vol. I, $\mathrm{n}^{\circ}$ 3, 1948, p. 8-11.

${ }^{8}$ English Masques, p. 227.

${ }^{9}$ Ibid., p. 237.
} 
all as rich as might be, but the strangeness of the habits was most admired ${ }^{10}$.

Tous ces changements indiquent une profonde métamorphose du masque sous l'influence française. L'insistance sur le caractère spectaculaire du masque au détriment du contenu littéraire et de la trame dramatique, la généralisation des entrées dans l'antimasque, marquent une érosion profonde de l'idéal jonsonien, notamment de son ambition littéraire : comme en France, et sous l'influence du ballet à entrées, le divertissement spectaculaire l'emporte sur l'idéal humaniste de l'œuvre unissant poésie, musique et danse.

\section{Masque et influence française dans le théâtre caroléen}

Si le théâtre reflète les influences françaises, quel peut être le mode d'insertion dans un cadre dramatique d'un masque devenu sans aucun intérêt dramatique? La question se pose d'autant plus que masque et théâtre suivent des évolutions parallèles, avec une tendance croissante au spectaculaire. Ainsi, Shirley, le librettiste, transpose-t-il à la scène (dans Changes) le problème qui se pose au même moment au masque - l'évolution vers davantage de spectaculaire, et moins d'intérêt dramatique. Il est également intéressant de constater que, pour Shirley, le masque peut être l'instrument de cette évolution. En effet, dans la comédie Changes, lors de la préparation d'un masque, le commanditaire se plaint de ce qu'une comédie ne peut plus se passer de divertissements dansés, et même que «your dance is the best language of some Comedies », ce qui n'est pas seulement une critique du mauvais théâtre, mais indique aussi que l'abus de danses est une cause de médiocrité dramatique. Caperwit marque bien l'évolution avec la pratique plus ancienne - élisabéthaine - de proposer une danse à la fin de la pièce, «a jig », pour le seul plaisir du spectateur et sans lien avec la pièce :

DANCER. A Maske will be delightful to the Ladies.

CAPERWIT. Oh Sir, what Playes are taking without these Pretty devices? Many Gentlemen Are not, as in the dayes of understanding, Now satisfied without a Jigge, which since They cannot, with their honor, call for, after

10 Ibid., p. 241. 
The play, they looke to be serv'd up ith'middle :

Your dance is the best language of some Comedies ${ }^{11}$.

Le nombre de masques (ou éléments de masque) introduits dans les comédies de Brome et de Shirley justifie à première vue les craintes de Caperwit. Richard Brome et James Shirley utilisent à la fois de nombreux éléments du masque, voire des masques présentés comme complets - bien qu'ils ne correspondent pas, en fait, à la structure du masque tel qu'il se représentait à la Cour ${ }^{12}$. Il est vrai que de tels épisodes existent déjà à l'époque élisabéthaine et jacobéenne (pour mémoire, le masque de Cérès dans The Tempest, ou le masque à l'acte v de Cynthia's Pleasures de Ben Jonson, masque d'Orphée dans The Mad Lover de Beaumont et Fletcher), mais pas avec cette fréquence, ni - sauf rares exceptions comme le masque d'Orphée dans The Mad Lover - avec un tel développement. À l'opposé, chez Richard Brome, l'insertion d'un masque ou d'éléments de masque est la norme : dans The Antipodes (masque de la Discorde); dans The English Moor (masque des Cornes); dans The Northern Lass; chez Shirley, on trouve des masques dans The Cardinal et Changes, pour n'en citer que quelques-uns parmi les plus intéressants.

L’importance et la fréquence de ces masques indique bien que l'esprit de promotion du spectaculaire à l'œuvre dans le masque sous l'influence française imprègne aussi le théâtre, et que le masque en est un agent essentiel. Ne pouvant faire ici l'analyse de toutes les comédies précédemment citées, j'ai choisi deux comédies de Brome, The English Moor et The Antipodes, représentatives de cette tendance.

Le masque des Cornes de The English Moore (I.iii) est clairement sous influence française, car il utilise un antimasque à entrées. Ce masque a pour sujet les mariages mal assortis, et commence par un discours de Mercure sur les dangers des mariages sans amour. Ce développement est illustré par une série d'entrées grotesques, «a drove of horn-beasts » comme le fait remarquer un personnage (I.iii.157) :

Enter the Masquers. A Lawyer with stagges hornes followed by a Courtier. A Countrey Chuffe with Rammes hornes, and a Souldier. An

\footnotetext{
11 J. Shirley, Changes, IV.391-399.

12 Voir l'introduction de M. Lefkowitz, in Trois masques à la cour de Charles I, Paris, Éditions du CNRS, 1976.
} 
Usurer with Goates Hornes, and a Scholler. A spruce Citizen with Oxe hornes, and a Butcher ${ }^{13}$.

Ces entrées sont simplement commentées, de façon souvent redondante, comme dans The Triumph of Peace : ici par Mercure, dont le commentaire redouble l'avertissement aux mauvais maris :

The first's a Lawyer, that by strife prevaild

To wed a wife, that was by Love intaild

To a Courtier ; who had the happe,

Soon after, to adorne him with that Cappe.

The next a Countrey Cormorant, whose great wealth,

By a bad Fathers will, obtaind, by stealth,

A valiant Souldiers Mistress ${ }^{14}$.

Le masque reflète donc l'influence du ballet de cour, au sein du théâtre. Mais avec une différence essentielle: ce masque à entrées est étroitement lié à l'intrigue. Ici, c'est un avertissement donné par les galants de la ville au vieil usurier Quicksands, qui doit épouser la jeune Millicent, laquelle y est forcée par son oncle. De plus, le masque s'intègre à la structure musicale de la pièce, car il sera complété par un second divertissement dansé à la scène iii de l'acte IV, qui n'est en fait qu'un stratagème pour duper Quicksands. Cette danse a elle aussi un caractère spectaculaire marqué : elle met en scène une danse de six Maures, en fait des personnages déguisés dont une actrice grimée en Maure. Cette danse est suivie de la pantomime grotesque d'un des personnages. Mais ce divertissement spectaculaire a aussi une fonction dramatique, puisque la danse n'est est en fait qu'une forme chorégraphiée de l'intrigue, tout comme le masque n'est autre qu'une intégration dramatique du principe des entrées.

Ce même principe est à l'œuvre dans The Antipodes, avec un degré supplémentaire d'intégration du masque à la structure dramatique. La comédie ne présente pas de masque à entrées comme tel, mais elle se sert du principe des entrées dérivé du masque comme principe structurel, cette fonction étant confirmée par le masque de l'acte $v$.

Dans The Antipodes, toute l'intrigue vise à guérir la folie d'un jeune homme, Peregrine. À cette fin, on va faire croire à Peregrine qu'il est transporté dans le monde parallèle mais inversé des Antipodes, un

\footnotetext{
${ }^{13}$ R. Brome, The English Moor, I.iii.152-156.

14 Ibid., I.iii.160-166.
} 
monde renversé qui est le reflet de sa propre folie. La présentation du monde renversé se fait à travers une série de personnages qui occupe la majeure partie de l'acte III et de l'acte IV. Chaque saynète montre les réflexions de Peregrine aux personnages qui défilent devant ses yeux. Toute cette partie tient à mes yeux de la pièce dans la pièce et du masque, car ce défilé de personnages, introduits par la musique des hautbois, semble venir tout droit des entrées à la française, par le traitement comme par le thème. Après un prologue, chacune de ces «entrées» est introduite et commentée par un personnage, exactement comme l'allégorie de la Fantaisie introduit les entrées de l'antimasque dans The Triumph of Peace (1634). De plus, le thème du monde renversé, de même que celui de la métamorphose, sont récurrents dans le ballet de cour ${ }^{15}$ :
III.ii : Enter Lawyer and Poet
III.iii : Enter a spruce young Captain
III.iv : Enter Buff Woman
III.v : Enter a Beggar and a Gallant
III.viii : Enter Gentleman and Officer
III.ix : Enter Citizen and Officer
IV.ii : Enter a young Gentleman, and an old Servingman
IV.iii : Enter Constable ad Watch
IV.v : Enter Women and Man-scold
IV.vi : Enter a Courtier (counting his money, and a second Courtier at a distance)
IV.viii : Enter Carman and Waterman

Comme dans les entrées, la succession des personnages dans de courtes scènes (III.ii à III.vi, puis III.viii, III.ix...), illustre de façon assez lâche le thème du monde renversé. Elle rejoint aussi la pratique des entrées en ce que ce défilé délimite de fait au sein de la pièce un espace où le spectaculaire et le didactique - montrer à Peregrine, à travers la folie des hommes, sa propre folie) l'emportent sur la progression dramatique.

Ce long épisode n'en est pas moins lié par la thématique de la pièce au masque qui célèbre le retour de Peregrine à la raison (IV.xi, v.xi, V.xii). Y apparaissent la Discorde, la Jalousie, la Mélancolie. Après l'air d'introduction de la Discorde ( Song in Untunable Notes », v.xi) a lieu une danse des allégories de la Discorde. L'Harmonie s'avance

\footnotetext{
15 Ainsi dans le Ballet du Monde Renversé (1630) où l'on voit « une femme qui bat son mary », « un aveugle conduisant un clairvoyant ». Voir P. Lacroix, op. cit., vol. 6.
} 
ensuite, suivie de Mercure, Bacchus, Apollon, et chasse la Discorde et ses acolytes. La danse du groupe de la Discorde met en valeur l'entrée ordonnée du groupe de l'harmonie, contraste qui est renforcé par l'air de l'Harmonie, qui répond de façon symétrique à celui de la Discorde.

Ce masque est ainsi plus proche du modèle jonsonien que des derniers masques caroléens, notamment par l'introduction de l'antimasque de la Discorde, qui met en valeur par contraste le retour de l'Harmonie. Le masque résume tout le mouvement de la pièce, y compris les scènes à entrées successives des actes III et IV. Si donc imprégnation il y a de la comédie par les structures du masque - en deux endroits au moins - et si cette présence du masque obéit à un souci de théâtralisation accrue, de grand spectacle, elle ne se fait pas au détriment de l'intérêt dramatique, sinon de façon passagère - il faut bien constater le passage d'une logique dramatique à une logique du spectacle, sans progression de l'intrigue, lors des entrées des actes III et IV. The Antipodes présente un recours important au masque et à ses structures, mais qui reste soumis à une logique dramatique, si importante que soit la participation de la musique à la comédie ${ }^{16}$. L'emprunt du masque devient ainsi un procédé dramatique à part entière, et même son côté spectaculaire contribue à souligner la progression de l'intrigue: les entrées des Antipodes mènent à la guérison du jeune Peregrine dans le cadre de l'intrigue de la pièce.

Dans The Antipodes comme dans The English Moor, l'utilisation même du principe des entrées opère donc dans un esprit très différent de celui de masques comme The Triumph of Peace ou de Salmacida Spolia. Le cadre dramatique réclame une cohérence entre le masque et l'intrigue principale.

L'influence du ballet de cour français modifie profondément le masque caroléen, et cette influence se retrouve dans le théâtre de Brome. Cette influence y est présente, mais gommée : car la décadence du masque jonsonien sous l'effet de l'influence française s'articule mal avec les besoins de la scène. L'insertion de masques témoigne d'une importance accrue du spectaculaire dans ces œuvres, ce qui est aussi un trait important de l'évolution sous influence du masque; mais l'influence du

\footnotetext{
${ }^{16}$ Pour une analyse complète de la pièce, il faudrait ajouter tous les épisodes musicaux tels que sonneries et chansons, qui contribuent à musicaliser l'ensemble de façon homogène et à renforcer le sentiment d'un divertissement hybride.
} 
ballet de cour (les entrées) provoque une dilution de l'intérêt et de l'unité dramatiques qui n'est pas compatible avec le développement cohérent de l'intrigue au théâtre. Ce peut être une des raisons pour lesquelles les masques de Brome semblent plutôt revenir au principe jonsonien d'une unité dramatique qui lie texte et musique.

L'utilisation du divertissement de cour dans ce théâtre ne permet pas de conclure à une quelconque décadence, au contraire de celle qui annonce le profond remaniement du masque à la Restauration. Au contraire, l'intégration du masque à la dynamique de l'intrigue mène à une intégration de l'élément musical à la structure dramatique au théâtre, alors qu'il n'est pas préservé dans le masque. Ainsi s'opère une hybridation bien plus réussie des genres ${ }^{17}$.

Du spectaculaire à la spéculation sur l'hybridation générique, l'utilisation du masque dans le théâtre de Brome approfondit un rapprochement entre musique et théâtre qui se poursuivra sous la Restauration. Sous influence française, sous influence mutuelle, masque et théâtre ne font peut-être encore sous Charles I ${ }^{\text {er }}$ que l'ébauche d'un genre nouveau : mais en tout cas «l'ébauche d'un grand spectacle $^{18} »$.

Claire BARDELMANN

Université de Metz

\footnotetext{
${ }^{17}$ Certaines pièces sont en bonne partie chantées tant la proportion d'épisodes musicaux est élevée : ainsi dans 2 St Patrick in Ireland, de Shirley, où le personnage du Barde ne s'exprime pratiquement qu'en chansons.

18 H. Prunières, op. cit. p. 247. Il existe une continuité de l'influence française ainsi que du théâtre caroléen sur le théâtre musical de la Restauration : voir M. Lefkowitz, "Shadwell and Locke's Psyche: the French Connection », Proceedings of the Royal Music Association, vol. LVI (1979-1980), p. 42-55.
} 